Series A

I. MATHEMATICA

501

\title{
A GREEN'S INEQUALITY FOR THE POWER MATRIX
}

BY

MENAHEM SCHIFFER and OLLI TAMMI

HELSIN KI 1971

S U O M A L A N E N T I E D E A A T EM I A

doi:10.5186/aasfm.1972.501 
Copyright (C) 1971 by

Academia Scientiarum Fennica

Communicated 13 September 1971 by Lauri Myrberg.

KESKUSKIRJAPAINO

HELS INKI 1971 


\section{Introduction}

Let $S\left(b_{1}\right)$ be the class of all regular analytic functions in the unit disk, which are univalent, satisfy the boundedness condition

$$
|f(z)|<1 \quad \text { in }|z|<1
$$

and have the Taylor series

$$
f(z)=\sum_{\nu=1}^{\infty} b_{\nu} z^{\nu}, \quad 0<b_{1} \leqq 1 .
$$

We denote

$$
a_{\nu}=b_{v} / b_{1}, \quad v=1,2, \ldots
$$

and wish to study the coefficient problem for this class.

We use an argument which is closely related to the classical area principle. Let $\Gamma_{r}$ be the image of the circle $|z|=r$ under the conformal mapping $w=f(z)$. This curve separates the origin from the unit periphery $|w|=1$ and we denote the ring domain bounded by $\Gamma_{r}$ and $|w|=1$ by $D_{r}$. If $g^{\prime}(w)$ is regular analytic in $D_{r}$ and has an integral with singlevalued real part, we may apply to it the Green's identity in the form

$$
0 \leqq \int_{D_{\mathbf{r}}}\left|g^{\prime}(w)\right|^{2} d \tau=\frac{1}{i} \int_{\partial D_{\boldsymbol{r}}} \operatorname{Re}\{g(w)\} g^{\prime}(w) d w .
$$

By an appropriate choice of $g^{\prime}(w)$ and use of Fourier relations one may then obtain useful estimations for the $a_{v}$. In [8] the function $g(w)$ was built up from the Faber polynomials of the function $f(z)$ and generalized Grunsky-Nehari inequalities were obtained. In accordance with the methods used in [2], [10] we will now apply a new function $g(w)$. This leads us to a different formalism for deriving coefficient inequalities which involve the power matrix of a function (2) instead of its Grunsky coefficients.

We define the Taylor (resp. Laurent) series

$$
f(z)^{n}=\sum_{k=n}^{\infty} c_{n k} z^{k} \quad \text { for } n \lessgtr 0
$$

and use the notation 


$$
\log \frac{f(z)}{z}=\sum_{k=0}^{\infty} c_{0^{k}} z^{k} .
$$

The double infinite triangular matrix $\left(\left(c_{n k}\right)\right)$ is, of course, closely related to the coefficient schemes which occur in the Grunsky inequalities. But it is surprising to see how elegant inequalities can be formulated and proved in terms of this power matrix. This matrix has already been studied by various authors and has many useful properties [3], [6], [8], [9].

\section{The basic inequalities}

Our new choice is the test function

$$
g(w)=x_{0} \log w+\sum_{\substack{n=-N \\ n \neq 0}}^{N} x_{n} w^{n}, \quad x_{0}=\text { real, }
$$

which satisfies all requirements made for $g(w)$ to justify the identity (4).

Next we use the definitions (5) and (6) to transplant $g(w)$ into the unit disk where it has the Laurent development

$$
g[f(z)]=x_{0} \log z+\sum_{n=-N}^{N} \sum_{k=n}^{\infty} x_{n} c_{n k} z^{k} .
$$

We simplify the notation by introducing the new vector

$$
y_{k}=\sum_{n=-N}^{N} x_{n} c_{n k}, \quad k \geqq-N,
$$

and by writing

$$
g[f(z)]=x_{0} \log z+\sum_{k=-N}^{\infty} y_{k} z^{k} .
$$

We express now the inequality (4) in the form

$$
I_{2} \leqq I_{1}
$$

with

$$
I_{1}=\frac{1}{i} \oint_{|w|=1} \operatorname{Re}\{g(w)\} d g(w)
$$

and

$$
\begin{aligned}
I_{2} & =\frac{1}{i} \int_{\Gamma_{r}} \operatorname{Re}\{g(w)\} d g(w) \\
& =\frac{1}{i} \int_{|z|=r} \operatorname{Re}[g(f)] d g(f) .
\end{aligned}
$$


The first integral is immediately evaluated by use of the orthonormality relations

$$
\frac{1}{2 \pi i} \int_{|w|=1} w^{-k} w^{l} \frac{d w}{w}=\delta_{k l}
$$

We obtains, because of $\bar{w}=w^{-1}$ on $|w|=1$,

$$
I_{1}=\frac{1}{2 i} \oint_{\substack{|w| \\ \sum_{n=1-N}}}^{N}\left(x_{n} w^{n}+\dot{\bar{x}}_{n} w^{-n}\right)\left(x_{0}+\sum_{\substack{m=-N \\ m \neq 0}}^{N} x_{m} m w^{m}\right) \frac{d w}{w}=\pi \sum_{-N}^{N} n\left|x_{n}\right|^{2}
$$

Similarly, we can use the orthonormality relations corresponding to (12) to evaluate the integral (11). The Laurent series $\left(8^{\prime}\right)$ converges absolutely and uniformly on $|z|=r$ and we have here $\bar{z}=r^{2} z^{-1}$. Hence

$$
\begin{aligned}
I_{2} & =\frac{1}{2 i} \oint_{|z|=r}\left[2 x_{0} \log r+\sum_{k=-N}^{\infty}\left(y_{k} z^{k}+\bar{y}_{k} r^{2 k} z^{-k}\right)\right] \cdot\left[x_{0}+\sum_{l=-N}^{\infty} l y_{l} z^{l}\right] \frac{d z}{z} \\
& =\pi\left[2 x_{0}^{2} \log r+2 x_{0} \operatorname{Re}\left\{y_{0}\right\}+\sum_{l=-N}^{\infty} l\left|y_{l}\right|^{2} r^{-2 l}\right] .
\end{aligned}
$$

Therefore (4) and (9) lead to the inequality

$$
2 x_{0}^{2} \log r+2 x_{0} \operatorname{R} \vartheta\left\{y_{0}\right\}+\sum_{l=-N}^{N} l\left|y_{l}\right|^{2} r^{-2 l} \leqq \sum_{n=-N}^{N} n\left|x_{n}\right|^{2} .
$$

The result simplifies if we let $r \rightarrow 1$. We obtain

$$
2 x_{0} \operatorname{Re}\left\{y_{0}\right\}+\sum_{l=-N}^{N} l\left|y_{l}\right|^{2} \leqq \sum_{n=-N}^{N} n\left|x_{n}\right|^{2}
$$

This is a remarkable property of the linear transformation (9) of the quite arbitrary complex vector $\left\{x_{n}\right\}$.

We state the following weakened rersion of (16) as

Theorem. For all functions of the class $S\left(b_{1}\right)$ we have the inequality

$$
2 x_{0} \operatorname{Re}\left\{y_{0}\right\}+\left.\sum_{l=-N}^{N} l\left|y_{l}{ }^{2} \leqq \sum_{l=-N}^{N} l\right| x_{l}\right|^{2}
$$

and equality holds if and only if $y_{v}=0$ for $v>N$. The numbers $y_{l}$ are defined by the linear transformation (9) of the vector $\left\{x_{\boldsymbol{l}}\right\}$, which is quite arbitrary except for the restriction $x_{0}=$ real.

\section{Löwner's differential equation and the power matrix}

We have been led in a natural way to introduce the linear transformation (9) of an arbitrary complex $(2 N+1)$-vector $\left\{x_{n}\right\}$ by means of the power 
matrix of a function of the class $S\left(b_{1}\right)$. It is very instructive to discuss this result from the point of view of the Löwner theory.

Let

$$
f(z, t)=c^{-t} \sum_{\nu=1}^{\infty} a_{\nu}(t) z^{\nu}
$$

be a solution of Löwner's differential equation

$$
\frac{\partial f}{\partial t}=-z \frac{1+x z}{1-x z} \frac{\partial f}{\partial z}
$$

with the initial condition

$$
f(z, 0)=z
$$

For given value $t, f(z, t)$ belongs always to the class $S\left(e^{-t}\right)$. The main subclass of the Löwner functions consists of mappings of the unit disk onto the unit disk slit along an arc which grows with increasing values of $t$.

Observe now that

$$
f(z, t)^{n}=\sum_{k=n}^{\infty} c_{n k}(t) z^{k}, \quad n \lesseqgtr 0,
$$

and

$$
\log \frac{f(z)}{z}=\sum_{k=0}^{\infty} c_{0 k}(t) z^{k}
$$

define the variable power matrix

$$
C(t)=\left(\left(c_{n k}(t)\right)\right) .
$$

Since we have for every differentiable function $F(f)$ the same differential equation

$$
\frac{\partial}{\partial t} F[f(z, t)]=-z \frac{1+x z}{1-x z} \frac{\partial}{\partial z} F[f(z, t)]
$$

we can derive the identities

$$
\sum_{k=n}^{\infty} \dot{c}_{n k}(t) z^{k}=-\left(1+2 \sum_{\varrho=1}^{\infty} x^{g} z^{\varrho}\right) \sum_{k=n}^{\infty} k c_{n k}(t) z^{k}
$$

if $n \neq 0$ and

$$
\sum_{k=0}^{\infty} \dot{c}_{0 k}(t) z^{k}=-\left(1+2 \sum_{\varrho=1}^{\infty} x^{\varrho} z^{\varrho}\right)\left(1+\sum_{k=1}^{\infty} k c_{0 k}(t) z^{k}\right) .
$$

Here - denotes differentiation with respect to $t$. Comparison of coefficients yields then for $n \neq 0$ 


$$
\dot{c}_{n k}(t)=-k c_{n k}(t)-2 \sum_{\varrho=1}^{\infty} \varkappa^{\varrho}(k-\varrho) c_{n(k-\varrho)}(t)
$$

and for $k>0$

$$
\dot{c}_{0 k}(t)=-k c_{0 k}(t)-2 \sum_{\varrho=1}^{\infty} \varkappa^{\varrho}(k-\varrho) c_{0(k-\varrho)}(t)-2 \varkappa^{k} .
$$

The sums in the preceding formulas are finite since $c_{n m}=0$ if $n>m$. Finally, we have

$$
\dot{c}_{00}(t)=-1 \text {. }
$$

We define now the variable vector

$$
y_{k}(t)=\sum_{n=-N}^{N} x_{n} c_{n k}(t)
$$

whose significance has become evident by our results in Section 2. We use (27), (28) and (29) to obtain

$$
\begin{gathered}
\dot{y}_{k}(t)=-k y_{k}(t)-2 \sum_{\varrho=1}^{\infty}(k-\varrho) x^{\varrho} y_{k-\varrho}(t) \quad \text { for } k<0 \\
\dot{y}_{0}(t)=2 \sum_{\varrho=1}^{\infty} \varrho x^{\varrho} y_{-\varrho}(t)-x_{0},
\end{gathered}
$$

(33) $\quad \dot{y}_{k}(t)=-k y_{k}(t)-2 \sum_{\varrho=1}^{\infty}(k-\varrho) x^{\varrho} y_{k-\varrho}(t)-2 x_{0} x^{i k} \quad$ for $k>0$.

We define, of course, $y_{-v}(t) \equiv 0$ for $v>N$.

Next, we form the expression

$$
\sum_{k=-N}^{N} k \bar{y}_{k} \dot{y}_{k}=-\sum_{k=-N}^{N} k^{2}\left|y_{k}\right|^{2}-2 \sum_{\varrho, k} k(k-\varrho) x^{\varrho} \bar{y}_{k} y_{k-\varrho}-2 x_{0} \sum_{k=1}^{N} k x^{k} \bar{y}_{k} .
$$

Let $l=k-\varrho$ and write $(34)$ as

(35) $\sum_{k=-N}^{N} k \bar{y}_{k} \dot{y}_{k}=-\sum_{k=-N}^{N} k^{2}\left|y_{k}\right|^{2}-\underset{\substack{k, l=-N \\ k>l}}{2} \sum_{\substack{k \\ k>l}}^{N} l \bar{y}_{k} y_{l} \varkappa^{k-l}-2 x_{0} \overline{z(t)}-x_{0}\left(\overline{\dot{y}_{0}}+x_{0}\right)$, where we introduce the notation

$$
z(t)=\sum_{k=-N}^{N} k x^{-k} y_{k}
$$

and use the identity (32). We add to the expression (35) its conjugate complex value and obtain

$$
\begin{aligned}
& \frac{d}{d t}\left[\sum_{k=-N}^{N} k\left|y_{k}(t)\right|^{2}+2 x_{0} \operatorname{Re}\left\{y_{0}(t)\right\}\right]=-2\left[|z(t)|^{2}+2 x_{0} \operatorname{Re}\{\bar{z}\}+x_{0}^{2}\right] \\
& \quad=-2\left|z(t)+x_{0}\right|^{2}
\end{aligned}
$$


We have thus proved the following:

Theorem. The functional

$$
S(t)=2 x_{0} \operatorname{Re}\left\{y_{0}(t)\right\}+\sum_{k=-N}^{N} k\left|y_{k}(t)\right|^{2}
$$

is monotonously decreasing with the parameter value $t$, except in the case

$$
x_{0}+\sum_{k=-N}^{N} k x^{-k} y_{k}(t) \equiv 0 .
$$

Observe that the matrix $C(t)$ has for $t=0$ the values

$$
\left\{\begin{array}{l}
c_{n k}=\delta_{n k} \quad \text { for } n \neq 0 \\
c_{0 k}=0
\end{array}\right.
$$

Hence we find

$$
y_{k}(t)=x_{k} \quad \text { for } \quad k \neq 0, y_{0}(t) \equiv 0 .
$$

Thus the inequality (16) is obtained as a consequence of our more general result in the case of slit mappings and hence holds for the most general function of class $S\left(b_{1}\right)$.

Dr. G. Goodman has pointed out that the close relation between the Löwner differential equation and Julia's variational formula [4] allows the following generalization:

Theorem. Let $f(z)$ and $f^{*}(z)$ be univalent in $|z|<1$ and normalized by the conditions $f(0)=f^{*}(0)=0, f^{\prime}(0)>0, f^{* \prime}(0)>0$. Associate with each the functional

$$
S=2 x_{0} \operatorname{Re}\left\{y_{0}\right\}+\sum_{l=-N}^{N} l\left|y_{l}\right|^{2}, S^{*}=2 x_{0} \operatorname{Re}\left\{y_{0}^{*}\right\}+\sum_{l=-N}^{N} l\left|y_{l}^{*}\right|^{2},
$$

respectively. If $f(z)$ maps the unit disk onto the domain $D, f^{*}(z)$ maps onto $D^{*}$ and if $D \subset D^{*}$, then

$$
S \leqq S^{*} .
$$

The functional $S$ is therefore for fixed vector $\left\{x_{n}\right\}$ domainmonotonic.

Let us verify that in the case (39) we have by (32)

$$
\begin{aligned}
\dot{y}_{k}(t) & =-k y_{k}(t)-\varkappa^{k}\left(2 x_{0}+2 \sum_{l=-N}^{N} l \chi^{-l} y_{l}(t)\right) \\
& =-k y_{k}(t), \quad \text { if } \quad k>N .
\end{aligned}
$$

Since we suppose $y_{k}(0)=x_{k}=0$ for $k>N$, we see that in this case we have $y_{k}(t) \equiv 0$ for $k>N$ and the equivalence of the inequalities (16) and (17) is ensured. Thus, the study of the differential system (31), (32), 
$\left(33^{\prime}\right)$ with the condition (39) for $x$ leads to the characterization of the extremum functions for which equality holds in (17).

We can use the identity (37) to obtain finer estimates. For example, put in formula (33) $k=N$ to have

$$
\begin{aligned}
\dot{y}_{N}(t) & =-N y_{N}-2 \sum_{\varrho=1}^{2 N} x^{\varrho}(N-\varrho) y_{N-\varrho}-2 x_{0} x^{N} \\
& =-N y_{N}-2 x^{N} \sum_{\varrho=1}^{2 N} x^{\varrho-N}(N-\varrho) y_{N-\varrho}-2 x_{0} x^{N} \\
& =-N y_{N}-2 x^{N} \sum_{l=-N}^{N} l y_{l} x^{-l}+2 x^{N} y_{N} x^{-N}-2 x_{0} x^{N} \\
& =N y_{N}-2 x^{N}\left(x_{0}+\sum_{l=-N}^{N} l y_{l} x^{-l}\right) .
\end{aligned}
$$

Using the notation (35) we arrive at

$$
\frac{d}{d t}\left[e^{-N t} y_{N}(t)\right] e^{N t}=-2 x^{N}\left(x_{0}+z(t)\right)
$$

and using (37) and (38), we can write this result as

$$
\left|\frac{d}{d t}\left[e^{-N t} y_{N}(t)\right]\right|^{2} e^{2 N t}=-2 S^{\prime}(t) .
$$

We integrate between $t=0$ and $t=t_{0}$ :

$$
S(0)-S\left(t_{0}\right)=\frac{1}{2} \int_{0}^{t_{0}} c^{2 N t}\left|\frac{d}{d t}\left(e^{-N t} y_{N}(t)\right)\right|^{2} d t
$$

On the other hand, we apply the Schwarz inequality to

$$
\int_{0}^{t_{0}} \frac{d}{d t}\left[y_{N} e^{-N t}\right] d t=\int_{0}^{t_{0}} y_{N}(t) e^{-N t}
$$

to find

$$
\begin{aligned}
\mid y_{N}\left(t_{0}\right) e^{-N t_{0}} & -\left.x_{N}\right|^{2} \leqq \int_{0}^{t_{0}} e^{-2 N t} d t \int_{0}^{t_{0}} e^{2 N t}\left|\frac{d}{d t}\left[y_{N} e^{-N t}\right]\right|^{2} d t \\
& \leqq \frac{1-e^{-2 N t_{0}}}{N} \int_{0}^{t_{0}} e^{2 N t}\left|\frac{d}{d t}\left[y_{N} e^{-N t}\right]\right|^{2} d t
\end{aligned}
$$

Observe that $e^{-N t}=b_{1}(t)$; hence we can combine (47) and (49) to obtain 


$$
\sum_{l=-N}^{N} l\left|x_{l}\right|^{2}-\sum_{l=-N}^{N} l\left|y_{l}\right|^{2}-2 x_{0} \operatorname{R} \circlearrowright\left\{y_{0}\right\} \geqq \frac{N}{1-b_{1}^{2 N}}\left|b_{1}^{N} y_{N}-x_{N}\right|^{2} .
$$

Theorem. Define the functional

$$
T(t)=2 x_{0} \operatorname{Re}\left\{y_{0}\right\}+\sum_{l=-N}^{N} l\left|y_{l}\right|^{2}+\frac{N}{1-b_{1}^{2 N}}\left|b_{1}^{N} y_{N}-x_{N}\right|^{2} .
$$

We have the inequality

$$
T(t) \leqq T(0),
$$

which sharpens the estimate (17).

\section{Bilinear form of the basic inequality}

The inequality (17) is of elegant structure but is somewhat difficult to apply since the Hermitian forms on both sides are indefinite. It is therefore convenient to transform it into another set of inequalities which can be handled with greater ease. Let us write (17) as

$$
\left|x_{0}\right|^{2}+\left|y_{0}\right|^{2}+\sum_{l=1}^{N} l\left(\left|y_{l}\right|^{2}+\left|x_{-l}\right|^{2}\right) \leqq\left|x_{0}-y_{0}\right|^{2}+\sum_{l=1}^{N} l\left(\left|x_{l}\right|^{2}+\left|y_{-l}\right|^{2}\right) \text {. }
$$

Suppose now that we have $2 N+2$ complex numbers $H_{l}(l=0$, $1, \ldots, 2 N+1)$ which stand to $2 N+2$ other complex numbers $K_{l}$ $(l=0,1, \ldots, 2 N+1)$ in the relation

$$
\sum_{l=0}^{2 N+1}\left|H_{l}\right|^{2} \leqq \sum_{l=0}^{2 N+1}\left|K_{l}\right|^{2}
$$

We can use the Schwarz inequality and (54) to have

$$
\left|\sum_{=0}^{2 N+1} H_{l} K_{l}\right|^{2} \leqq \sum_{l=0}^{2 N+1}\left|H_{l}\right|^{2} \sum_{l=0}^{2 N+1}\left|K_{l}\right|^{2} \leqq\left(\sum_{l=0}^{2 N+1}\left|K_{l}\right|^{2}\right)^{2}
$$

and to infer

$$
\left|\sum_{l=0}^{2 N+1} H_{l} K_{l}\right| \leqq \sum_{l=0}^{2 N+1}\left|K_{l}\right|^{2}
$$

We use now the following values for $H_{l}$ and $K_{l}$ :

$$
\left\{\begin{array}{l}
H_{0}=x_{0}, H_{l}=\sqrt{l} y_{l}, H_{2 N-l+1}=\sqrt{l} x_{-l}, H_{2 N+1}=y_{0}, \\
K_{0}=x_{0}-y_{0}, K_{l}=\sqrt{l} y_{-l}, K_{2 N-l+1}=\sqrt{l} x_{l}, K_{2 N+1}=0 .
\end{array}\right.
$$

The inequality (53) coincides then with (54) and the consequent inequality (56) can be retranslated into 


$$
\begin{aligned}
& \left|x_{0}\left(x_{0}-y_{0}\right)+\sum_{l=1}^{N} l\left(y_{-l} y_{l}+x_{-l} x_{l}\right)\right| \\
& \leqq\left|x_{0}-y_{0}\right|^{2}+\sum_{l=1}^{N} l\left(\left|y_{-l}\right|^{2}+\left|x_{l}\right|^{2}\right)
\end{aligned}
$$

We rearrange to arrive at the

Theorem. The bilinear version of the basic inequality is

$$
\begin{array}{r}
\operatorname{Re}\left\{x_{0} y_{0}+\sum_{l=1}^{N} l\left(y_{-l} y_{l}+x_{-l} x_{l}\right)\right\} \\
\leqq\left|y_{0}\right|^{2}+\sum_{l=1}^{N} l\left(\left|y_{l-}\right|^{2}+\left|x_{l}\right|^{2}\right) .
\end{array}
$$

If $x_{0}$ or $\operatorname{Re}\left\{y_{0}\right\}$ vanish, we have the simpler version

$$
\operatorname{Re}\left\{\sum_{l=1}^{N} l\left(y_{-l} y_{l}+x_{-l} x_{l}\right)\right\} \leqq \sum_{l=1}^{N} l\left(\left|y_{-l}\right|^{2}+\left|x_{l}\right|^{2}\right) .
$$

\section{Comparison with the Grunsky-Nehari inequalities}

We have now to study the relation of our new estimates (17) and (59) to the well-known inequalities of Grunsky and Nehari [1], [5] which were somewhat sharpened by us [8]. Their derivation was also based on Green's formula and the area principle, but the choice of the test functions $g(w)$ was different. The basic tool in this alternative approach is the concept of the Faber polynomial which can be defined by the generating function

$$
-\log (1-t f(z))=\sum_{\nu=1}^{\infty} \frac{1}{v} F_{\nu}(t) z^{\nu}
$$

where $f(z)$ is any analytic function defined near the origin. One can easily see that $F_{v}(t)$ is a polynomial of degree $v$ in $t$ with the property that

$$
F_{\nu}\left(\frac{1}{f(z)}\right)=\frac{1}{z^{\nu}}+\sum_{g=0}^{\infty} \gamma_{\nu_{0}} z^{\prime \prime}
$$

has the principal part $1 / z^{\nu}$. To define the coefficients $\gamma_{\nu^{\circ}}$ in the development (61) let us introduce further the series derelopments

$$
\log \frac{f(z)-f(\zeta)}{z-\zeta}=\sum_{m, n=0}^{\infty} A_{m n} z^{m} \zeta^{n}
$$

and

$$
-\log (1-f(z) \overline{f(\zeta)})=\sum_{m, n=0}^{\infty} B_{m n} z^{m} \bar{\zeta}^{n}
$$


A. I. 501

It is clear that $\left(\left(A_{m n}\right)\right)$ is a symmetric matrix while $\left(\left(B_{m n}\right)\right)$ is hermitian. We can combine (62) with (61) and write

$$
\log \frac{f(z)}{z}+\log \left(1-\frac{f(\zeta)}{f(z)}\right)=\log \left(1-\frac{\zeta}{z}\right)+\sum_{m, n=0}^{\infty} A_{m n} z^{m} \zeta^{n},
$$

that is,

$$
\log \frac{f(z)}{z}-\sum_{n=1}^{\infty} \frac{1}{n} F_{n}\left(\frac{1}{f(z)}\right) \zeta^{n}=-\sum_{n=1}^{\infty} \frac{1}{n} \frac{\zeta^{n}}{z^{m}}+\sum_{m, n=0}^{\infty} A_{m n} z^{m} \zeta^{n} .
$$

This leads by comparison of coefficients to

$$
F_{n}\left(\frac{1}{f(z)}\right)=\frac{1}{z^{n}}-\sum_{m=0}^{\infty} n A_{m n} z^{m}
$$

and

$$
\log \frac{f(z)}{z}=\sum_{m=0}^{\infty} A_{m 0} z^{m} .
$$

Similarly, we can apply the same argument to (63) and find

$$
\frac{1}{n} \bar{F}_{n}(f(z))=\sum_{m=0}^{\infty} B_{m n} z^{m} .
$$

Suppose now we choose as test function $\tilde{g}(w)$ the aggregate

$$
\left\{\begin{array}{l}
\tilde{g}(w)=x_{0} \log w+\sum_{l=1}^{N}\left[\bar{\xi}_{l} \bar{F}_{l}(w)-\xi_{l} F_{l}\left(\frac{1}{w}\right)\right], \\
x_{0}=\text { real. }
\end{array}\right.
$$

The advantage of this choice lies in the fact that we have

$$
\operatorname{Re}\{\tilde{g}(w)\}=0 \text { on }|w|=1 .
$$

Hence, in the integration over $\partial D_{r}$ we have only to consider the boundary curve $\Gamma_{r}$ defined in Section 1 . However, we have seen that in a more general choice of $g(w)$ the integration over the circle $|w|=1$ can be carried out easily and does not create any difficulty. By means of (65), (66) and (67), we can express

$$
\tilde{g}(f(z))=x_{0} \log z-\sum_{l=1}^{N} \xi_{l} z^{-l}+\sum_{m=0}^{\infty} C_{m} z^{m}
$$

with

$$
C_{m}=A_{m 0} x_{0}+\sum_{l=1}^{N} l\left(A_{m l \xi l}+B_{m l \xi} \bar{\xi}_{l}\right)
$$


A simple use of the Fourier relations for the powers of $z$ on $|z|=r$ and passage to the limit $r=1$ leads from (4) to the estimate

$$
\sum_{l=1}^{N} l\left|C_{l}\right|^{2} \leqq \sum_{l=1}^{\infty} l\left|C_{l}\right|^{2} \leqq \sum_{l=1}^{N} l\left|\xi_{l}\right|^{2}-2 x_{0} \operatorname{Re}\left\{C_{0}\right\}
$$

Let us choose $x_{0}$ and the $\xi_{l}$ such that $x_{0} \operatorname{Re}\left\{C_{0}\right\}=0$. The method of bilinearization used in Section 4 yields then

$$
\operatorname{Re}\left\{\sum_{l=1}^{N} l \xi_{l} C_{l}\right\} \leqq \sum_{l=1}^{N} l\left|\xi_{l}\right|^{2}
$$

and the use of (71) and the substitution $t_{l}=l \xi_{l}$ give

$$
\operatorname{Re}\left\{\sum_{m, l=1}^{N}\left(A_{m l} t_{m} t_{l}+B_{m l} t_{m} \bar{t}_{l}\right)\right\} \leqq \sum_{l=1}^{N} \frac{\left|t_{l}\right|^{2}}{l},
$$

the Grunsky-Nehari inequalities in generalized form.

This analysis shows that the Grunsky-Nehari inequalities must be a special case of our inequalities because they have been obtained by a particular choice of a function of $w$ composed of one logarithm term and a rational function with $N$-th order poles at zero and infinity. However, while we can admit $2 N$ complex parameters and one real parameter $x_{0}$, the Grunsky-Nehari inequalities deal only with $N$ complex parameters.

To characterize the subclass of functions $\tilde{g}(w)$ defined by (68) within the general class of functions $g(w)$ defined by (8), we have to study the solution between the power matrix $\left(\left(c_{n k}\right)\right)$ and the matrices $\left(\left(A_{n k}\right)\right)$ and $\left(\left(B_{n k}\right)\right)$. We introduce for this purpose the additional matrix $\left(\left(P_{n k}\right)\right)$ by the equation

$$
F_{n}(t)=\sum_{k=1}^{n} P_{k n} t^{k}
$$

for the $n$-th Faber polynomial. $\left(\left(P_{n k}\right)\right)$ is an infinite triangular matrix. From (5) and (65), we have

$$
\begin{aligned}
F_{n}\left(\frac{1}{f(z)}\right) & =\sum_{k=1}^{n} \sum_{l=-k}^{\infty} P_{n k} c_{-k l} z^{l} \\
& =\frac{1}{z^{n}}-\sum_{l=0}^{\infty} n A_{l n} z^{l} .
\end{aligned}
$$

Hence,

$$
\sum_{k=1}^{n} P_{n k} c_{-k-l}=\delta_{n l}, \quad l \geqq 1,
$$

and 


$$
\sum_{k=1}^{n} P_{n k} c_{-k l}=-n A_{l n}, \quad l \geqq 0 .
$$

Similarly, we obtain from (5) and (67)

$$
\bar{F}_{n}(f(z))=\sum_{k=1}^{n} \sum_{l=k}^{\infty} \bar{P}_{n k} c_{k l} z^{l}=\sum_{l=1}^{\infty} n B_{l n} z^{l}
$$

whence

$$
\sum_{k=1}^{n} \bar{P}_{n k} c_{k l}=n B_{l n}
$$

Finally, comparison of (6) with (66) leads to

$$
c_{0 l}=A_{l 0} \text {. }
$$

Now we can express transforms $C_{m}$ of the vector $x_{0}, \xi_{1}$ defined by (71) as follows:

$$
C_{m}=c_{0 l} x_{0}+\sum_{k=1}^{N} x_{k} c_{k m}-\sum_{k=1}^{N} \bar{x}_{k} c_{-k m}
$$

with

$$
\bar{x}_{k}=\sum_{l=1}^{N} P_{l k} \xi_{l}, \quad k=1,2, \ldots, N
$$

Thus, given the vector $x_{0}, \xi_{l}(l=1, \ldots, N)$, let us associate with it the vector $x_{0}, x_{l}, x_{-l}(l=1, \ldots, N)$, where $x_{1}$ is defined by (81) and

$$
x_{-l}=-\bar{x}_{l} .
$$

With this notation we can write $(80)$ in the form

$$
C_{m}=\sum_{k=-N}^{N} x_{k} c_{k m}
$$

We see that for this special $x$-vector the numbers $C_{m}$ coincide with the transforms $y_{m}$ defined by (9). But observe that the index $m$ is supposed to be positive. On the other hand, a comparison of the development $\left(8^{\prime}\right)$ of $g(w)$ with the development $(70)$ of $\tilde{g}(w)$ leads to the solutions

$$
y_{-k}=-\xi_{k}, \quad k=1,2, \ldots, N \text {. }
$$

Finally, we easily verify the equation

$$
C_{0}=y_{0}
$$

Thus, with this choice of parameters $x_{k}$ we can express the inequality (17) in the form 


$$
2 x_{0} \operatorname{Re}\left\{C_{0}\right\}+\sum_{l=-N}^{N} l\left|y_{l}\right| \leqq \sum_{l=-N}^{N} l\left|x_{l}\right|^{2}=0,
$$

i.e.,

$$
2 x_{0} \operatorname{Re}\left\{C_{0}\right\}+\sum_{l=1}^{N} l\left|C_{l}\right|^{2} \leqq \sum_{l=1}^{N} l\left|\xi_{l}\right|^{2} .
$$

This is precisely the inequality (72).

It is then evident that the bilinear form (74) is a particular case of (59)

if $x_{-l}=-\bar{x}_{l}$.

\section{Stanford University}

Stanford, California, U.S.A.

University of Helsinki

Helsinki, Finland.

\section{References}

1. Grunsky, H., Koeffizientenbedingungen für schlicht abbildende meromorphe Funktionen.Math. Z. 45 (1939), 29-61.

2. Hummel, J., Schiffer, M., Coefficient inequalities for Bieberbach-Eilenberg functions - Arch. Rational Mech. Anal. (to appear).

3. JABOTINSKY, E., Representation of functions by matrices. Application to Faber polynomials - Proc. Amer. Math. Soc. 4 (1953), 546- 553.

4. Julia, G., Sur une équation aux dérivées fonctionelles liée à la représentation conforme - Ann. Sci. École Norm. Sup. (3) 39 (1922), 1-28.

5. Nehari, Z., Some inequalities in the theory of functions - Trans. Amer. Math. Soc. 75 (1953), $256-286$.

6. Schur, J., On Faber polynomials - Amer. J. Math. 67 (1945), 33-41.

7. Schiffer, M., Faber polynomials in the theory of univalent functions - Bull. Amer. Math. Soc. 54 (1948), 503-517.

8. - - - and TAмri, O., On the coefficient problem for bounded univalent functions - Trans. Amer. Math. Soc. 140 (1969), 461-474.

9. Tammi, O., Grunsky type of inequalities and determination of the totality of the extremal functions - Ann. Acad. Sci. Fenn. Ser. AI n:o 443 (1969), 1-20.

10. -»- On Green's inequalities for the third coefficient of bounded univalent functions - Ibid. n:o 481 (1970), 1-10. 